\title{
EMOCIONES POSITIVAS: UNA HERRAMIENTA PSICOLÓGICA QUE CONTRIBUYE AL PROCESO DE RESILIENCIA EN LOS PROFESIONALES DE LA EDUCACIÓN
}

\author{
Vicente de Vera García M. Inmaculada \\ Universidad Pública de Navarra (UPNA) \\ Departamento Ciencias Humanas y de la Educación \\ ivicentedevera@yahoo.es \\ Gabari Gambarte M.Inés \\ Universidad Pública de Navarra (UPNA) \\ Departamento Ciencias Humanas y de la Educación
}

Fecha de Recepción: 5 Marzo 2019

Fecha de Admisión: 1 Junio 2019

\section{RESUMEN}

El fenómeno de la resiliencia cobra especial importancia en el proceso educativo pues la escuela es un ambiente clave fundamental para adquirir competencias necesarias para salir adelante, gracias a la capacidad de las personas para sobreponerse ante las dificultades de la vida. Mediante estudio empírico, se valora el nivel de resiliencia expresado por los docentes participantes en relación con variables sociodemográficas y sociolaborales. La muestra invitada es el profesorado de Educación Secundaria de los 24 Institutos públicos de la provincia de Huesca ( $N=1.268)$ pertenecientes a la red pública de enseñanza en el curso 2014/15. Mediante participación voluntaria, se conforma la muestra definitiva con $n=167$ docentes (13.17\%). La resiliencia se evalúa mediante el Cuestionario de Evaluación de Resiliencia de Serrano-Martínez (2010). Los resultados confirman que las emociones positivas mitigan la vulnerabilidad a los estresores laborales favoreciendo el surgimiento de la resiliencia. Asimismo, la evidencia de resiliencia del colectivo encuestado se acrecienta con las condiciones vitales que aportan estabilidad afectivo-emocional y laboral.

Palabras clave: resiliencia; emociones positivas; docente; variables sociodemográficas; variables sociolaborales

\section{ABSTRACT}

Positive emotions: a psychological tool that contributes to the resilience process in education professionals. The phenomenon of resilience takes on special importance in the educational process, because school is a fundamental key environment for acquiring the necessary skills to get ahead thanks to the ability of people to overcome the difficulties of life. Through empirical study, the level of resilience expressed by participating teachers in relation to sociodemographic and socio- 


\section{EMOCIONES POSITIVAS: UNA HERRAMIENTA PSICOLÓGICA QUE CONTRIBUYE AL PROCESO DE RESILIENCIA EN LOS PROFESIONALES DE LA EDUCACIÓN}

labor variables is assessed. The invited sample is the the high school teachers of the 24 Institutes of the province of Huesca ( $N=1.268)$ belonging to the public teaching network in the $2014 / 15$ academic year. Through voluntary participation, the final sample is formed with $n=167$ teachers $(13.17 \%)$. Resilience is evaluated through the Resilience Evaluation Questionnaire of SerranoMartínez (2010). The results confirm that positive emotions mitigate vulnerability to job stressors favoring the emergence of resilience. Likewise, the evidence of resilience of the surveyed group increases with the vital conditions that provide affective-emotional and labor stability.

Keywords: resilience; positive emotions; teachers; sociodemographic variables; socio-labor variables

\section{INTRODUCCIÓN}

La resiliencia o capacidad para superar situaciones adversas 0 estresantes y salir fortalecido, logrando alcanzar una adaptación positiva Masten (2016), representa una de las principales dimensiones asociadas al bienestar psicológico y al éxito académico (Kristjánsson, 2012). El transcurso de adaptación puede ser inferido a partir del nivel de ajuste en diversos ámbitos sociales (familiar, escolar, etc.), por lo que no denota una invulnerabilidad al estrés, sino persistencia de las competencias funcionales y adaptativas mediante el empleo de estrategias de afrontamiento eficaces (González, Torres y Carreres, 2017; Félix, García y Mercado ibarra, 2018). La resiliencia forma parte del desarrollo evolutivo ordinario del ser humano (Gómez y Cavaco, 2016).

La acepción de resiliente reconoce el dolor, la lucha y el sufrimiento implícitos en el proceso, implica un considerable estrés y malestar emocional, a pesar del cual las personas sacan la fuerza que les permite seguir con sus vidas frente la adversidad o la tragedia (Morales, 2017). El proceso de ser y llegar a ser resiliente se va desarrollando de manera diferencial, según se suceden las etapas vitales y se van presentando las diferentes condiciones ambientales y laborales (Justo, 2010; Díaz y Barra, 2017). Algunos sujetos son más vulnerables que otros a los estresores, dependiendo de las estrategias cognitivas que se tengan para resolver los problemas, del tipo de ejercicio profesional y del desarrollo de factores de resistencia o de protección ligados a las variables individuales, como el tipo de personalidad y atributos o rasgos de la misma (Albar et al., 2004; Álvarez y Cáceres, 2010; Contreras y Peña, 2016; Carrara, 2018).

En el ámbito educativo, la resiliencia se refiere al potencial que tiene cada persona (docentes/discentes...). Los autores que exploran la resiliencia, destacan que algunas de las características de las personas resilientes están relacionadas con la flexibilidad cognitiva, la capacidad creativa, la habilidad para resolver problemas interpersonales, el buen nivel de autoconcepto y los vínculos de apego que han forjado en la infancia (Saavedra y Villalta, 2008; Ornelas, 2016).

Otro constructo que ha adquirido especial relevancia en los últimos años, entendido como un factor que contribuye al bienestar psicológico, es el optimismo (Knowlden, Hackman y Sharma, 2016). Algunos autores, como McKergow (2009) y Sttrata et al. (2013) y Franco (2017) subrayan el peso que emociones como el optimismo, asertividad y la autoestima tienen en la construcción del proceso resiliente. Se ha descrito, que los sujetos optimistas presentan altos niveles de resiliencia (Dawson y Pooley, 2013), lo que demuestra la estrecha relación que existe entre las emociones positivas y la resiliencia (Martínez y Ruch, 2017).

Asimismo, Carver y Scheier (2014), Oriol, Mendoza, Covarrubias y Molina (2017) y Serrano (2018), entre otros, refieren que una actitud optimista ante situaciones retadoras se relaciona con la confianza y la persistencia en la conducta aunque las cosas se pongan difíciles. En el mismo sentido, Pulido y Herrera (2018) corroboran que el optimismo ejerce una influencia diferencial en la valoración y afrontamiento de las dificultades, en el desenvolvimiento en el mundo social y acadé- 
mico y en el bienestar psíquico y físico y, señalan, que optimismo y pesimismo correlacionan de manera significativa con la autoeficacia y con desgaste profesional de manera diversa. Por tanto, dar un significado positivo a los eventos de la vida y tener un estilo de afrontamiento focalizado en el problema, ayuda a generar emociones positivas cuando se experimentan situaciones adversas (Alarcón, 2018; Vicente de Vera y Gabari, 2019).

\section{OBJETIVO}

Se pretende valorar el nivel de resiliencia expresado por docentes de Educación Secundaria de la provincia de Huesca (España) y analizar su relación con variables sociodemográficas y con variables de tipo sociolaboral.

\section{MATERIAL Y MÉTODOS}

Se plantea un estudio exploratorio de diseño transversal, descriptivo y analítico. La variable independiente es la pertenencia a un colectivo docente de Enseñanza Secundaria de la provincia de Huesca (España). La variable dependiente, los niveles de resiliencia percibidos en sus diferentes dimensiones en función del sexo, edad, estado civil, descendencia, tipo de contrato y antigüedad en el puesto.

\section{Participantes}

Los Equipos Directivos de los Centros de los Institutos de Enseñanza Secundaria ( $N=24$ I.E.S.) de la Red Pública de la provincia de Huesca contribuyen a la difusión del Cuestionario entre los Claustros. El criterio de inclusión en la muestra es la participación libre, voluntaria y anónima del profesorado sin identificación del centro educativo al que pertenece cada docente. La muestra aceptante la forman 167 docentes.

\section{Instrumentos}

La resiliencia se evalúa mediante el Cuestionario de Evaluación de Resiliencia de Serrano Martínez (2010), con 34 cuestiones estructuradas en una escala tipo Likert, con cinco niveles de repuesta desde nunca (1) a siempre (5). La evaluación psicométrica del cuestionario evidencia 8 ítems como inconsistentes, por lo que son eliminados. El análisis factorial exploratorio obtiene cinco factores: sensibilidad o afrontamiento emocional (6 ítems), superación o afrontamiento conductual (8 ítems), positivismo u optimismo ingenuo (6 ítems), pensamiento estereotipado (4 ítems) y tolerancia a la frustración (4 ítems).

El Factor I es el de sensibilidad o afrontamiento emocional con estrategias como el autocontrol, distanciamiento, revaluación positiva, autoinculpación y escape/evitación. El Factor II, es la superación 0 afrontamiento conductual, que mide la tendencia a pensar automáticamente en términos que faciliten una acción eficaz. El Factor III, recoge aspectos que aluden al positivismo u optimismo ingenuo y se refiere a la capacidad para pensar favorablemente, sabiendo enfatizar el lado positivo de la situación. El Factor IV, o pensamiento estereotipado, es la tendencia de algunas personas a dejarse llevar por las ideas establecidas socialmente. Por último, el Factor V de tolerancia a la frustración mide la capacidad para afrontar los problemas y limitaciones que nos encontramos en la vida, a pesar las molestias o incomodidades que nos causan.

La información sociodemográfica se recoge en cuatro variables (sexo, rango de edad, estado civil y número de hijos/as) y la sociolaboral en otras dos (antigüedad y estabilidad: contratado/a, funcionario/a, interino/a o sustituto/a). 


\section{EMOCIONES POSITIVAS: UNA HERRAMIENTA PSICOLÓGICA QUE CONTRIBUYE AL PROCESO DE RESILIENCIA EN LOS PROFESIONALES DE LA EDUCACIÓN}

\section{Análisis de datos}

Para el análisis estadístico descriptivo y el análisis de contingencias con Chi-cuadrado de Pearson se emplea el paquete estadístico SPSS versión 20 adoptando un nivel de significación estadística de $\mathrm{p}<, 05$.

\section{RESULTADOS}

La distribución de la muestra aceptante $(13,71 \%$ de la invitada) es: 94 mujeres $(56,29 \%)$ y 73 varones (43,71\%). El mayor porcentaje acumulado se sitúa entre los 46 y los 55 años $(38,92 \%)$. Predomina el profesorado con una experiencia de ejercicio docente de más de veinte años $(44,91 \%)$. Un alto porcentaje $(70,66 \%)$ son funcionarios/as. Asimismo, el 73,65\% de los docentes están casados/as o viviendo en pareja. En lo que se refiere a la descendencia, destaca la participación del profesorado con hijos/as. El 66,47\% del profesorado tiene algún hijo/a.

El cálculo del de Cronbach arroja una fiabilidad del cuestionario de ,815, por lo que se puede afirmar que los datos recogidos responden a las valoraciones pretendidas por el instrumento de medida. Los valores medios de cada factor se representan en la Figura 1.

Figura 1. Medias de los factores de la escala de resiliencia.

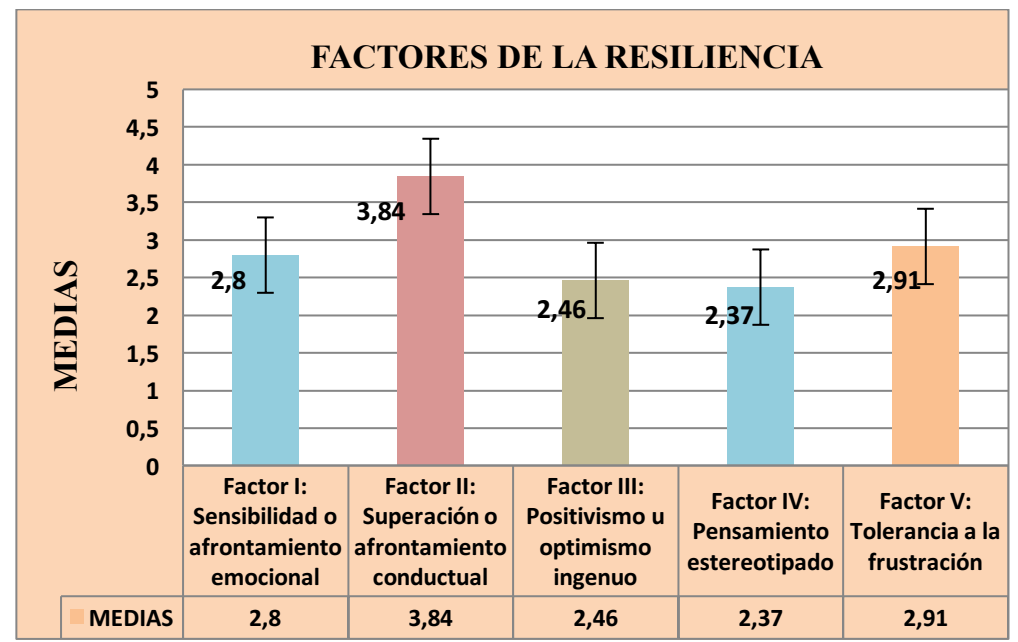

En la Tabla 1 se reflejan los percentiles de la muestra en relación con los diversos Factores de Resiliencia. 
Tabla 1. Evaluación resiliencia docentes secundaria de Huesca.

\begin{tabular}{|c|c|}
\hline Factores de resiliencia & $\begin{array}{r}\text { Docentes superan la mediana en cada } \\
\text { factor }\end{array}$ \\
\hline $\begin{array}{l}\text { Factor I: Sensibilidad o afrontamiento } \\
\text { emocional }\end{array}$ & $91(54.49 \%)$ \\
\hline $\begin{array}{l}\text { Factor II: Superación o afrontamiento } \\
\text { conductual }\end{array}$ & $88(52.69 \%)$ \\
\hline $\begin{array}{l}\text { Factor III: Positivismo u optimismo } \\
\text { ingenuo }\end{array}$ & $90(53.89 \%)$ \\
\hline Factor IV: Pensamiento estereotipado & $84(50.30 \%)$ \\
\hline Factor V: Tolerancia a la frustración & $88(52.69 \%)$ \\
\hline
\end{tabular}

Del total de la muestra, 22 participantes (13.17\%) obtienen valoraciones que superan la mediana en todos los factores de la escala y pueden considerarse resilientes. Según las variables sociodemográficas: a) en relación al sexo 13 son mujeres y 9 son hombres; b) respecto a edad, 9 tienen entre 46 y 55 años; 6 se sitúan en la franja de 56 a 65 años; 4 en la franja de 36 a 45 años y, por último, otros 2 sujetos oscilan entre los 22 a 35 años; b) según el estado civil 19 están casados/as 0 viviendo en pareja, 2 son solteros/as y uno/a no indica su estado civil; c) por su descendencia 14 tienen hijos/as.

Su distribución en función de las variables sociolaborales es la siguiente: a) según su acceso al puesto laboral 17 son funcionarios/as, y b) por su antigüedad en el desempeño profesional docente, 12 de ellos/as tienen una experiencia profesional de más de 20 años de ejercicio docente.

A partir del análisis de contingencias en relación con las variables sociodemográficas, según la variable sexo manifiestan mayor resiliencia las mujeres encontrando diferencias significativas en los ítems recogidos en la Tabla $2(p=, 001, p=, 012, p=, 043, p=, 025, p=, 005$ y $p=, 009)$. Según el rango de edad, los resultados significativamente superiores en ítems que indican resiliencia $(p=, 009$, $p=, 020$ y $p=, 025$ ) se obtienen en la franja de 46 a 55 años (ver Tabla 2). El profesorado casado manifiesta un grado significativamente mayor en resiliencia que el que no vive en pareja 0 está soltero en determinados ítems recogidos en la Tabla 2 ( $p=, 000, p=, 024, p=, 000, p=, 000, p=, 025, p=, 033$, $p=, 000$ y $p=, 002)$. Asimismo, se aprecian puntuaciones significativamente superiores en resiliencia en el profesorado con hijos en comparación con aquellos que no tienen descendencia $(p=, 01$, $p=, 028$ y $p=, 004)$. 
EMOCIONES POSITIVAS: UNA HERRAMIENTA PSICOLÓGICA QUE CONTRIBUYE AL PROCESO DE RESILIENCIA EN LOS PROFESIONALES DE LA EDUCACIÓN

Tabla 2. Resiliencia en relación a variables sociodemográficas en docentes de E. Secundaria de Huesca.

\begin{tabular}{|c|c|c|c|c|}
\hline $\begin{array}{c}\text { Variables } \\
\text { sociodemográficas }\end{array}$ & Ítem Resiliencia & Chi $^{2}$ & $\mathbf{p}$ & Diferencias \\
\hline \multirow{6}{*}{$\begin{array}{l}\text { Sexo: } \\
\text { seis ítems } \\
\text { con valor } \mathrm{p}<, 05\end{array}$} & $\begin{array}{l}\text { No soporto demasiado bien la } \\
\text { presión }\end{array}$ & 19,422 & ,001 & \multirow[t]{6}{*}{$q>0^{\lambda}$} \\
\hline & $\begin{array}{l}\text { Tras una discusión me siento } \\
\text { hundido y vacío }\end{array}$ & 12,897 & ,012 & \\
\hline & $\begin{array}{l}\text { Siento miedo de que los demás } \\
\text { me rechacen y me quede solo }\end{array}$ & 9,873 & ,043 & \\
\hline & $\begin{array}{l}\text { No me suelen afectar las críticas } \\
\text { ni positiva ni negativamente }\end{array}$ & 11,174 &, 025 & \\
\hline & $\begin{array}{l}\text { Me cuesta mucho reponerme de } \\
\text { una separación emocional }\end{array}$ & 14,942 &, 005 & \\
\hline & $\begin{array}{l}\text { Soy de los que piensa que en todo } \\
\text { lo malo hay algo bueno }\end{array}$ & 13,505 & ,009 & \\
\hline \multirow{3}{*}{$\begin{array}{l}\text { Edad: } \\
\text { tres ítems } \\
\text { con valor } \mathrm{p}<, 05\end{array}$} & Suelo llevar mal equivocarme & 26,592 & ,009 & \multirow{3}{*}{$\begin{array}{l}\text { Rango } 46- \\
55>36-45> \\
56-65>22-35\end{array}$} \\
\hline & $\begin{array}{l}\text { Siento miedo de que los demás } \\
\text { me rechacen y me quede solo }\end{array}$ & 24,126 &, 020 & \\
\hline & $\begin{array}{l}\text { Tiendo a exagerar los } \\
\text { acontecimientos negativos que me } \\
\text { ocurren }\end{array}$ & 23,293 & ,025 & \\
\hline \multirow{8}{*}{$\begin{array}{l}\text { Estado civil: } \\
\text { ocho ítems } \\
\text { con valor } \mathrm{p}<, 05\end{array}$} & Me preocupo en exceso & 47,994 &, 000 & \multirow{8}{*}{$\begin{array}{l}\text { Casado/ } \\
\text { viviendo en } \\
\text { pareja> } \\
\text { soltero>otras } \\
\text { opciones }\end{array}$} \\
\hline & $\begin{array}{l}\text { Considero que todas las personas } \\
\text { son buenas }\end{array}$ & 34,292 & ,024 & \\
\hline & $\begin{array}{l}\text { Realizo mis cometidos aunque } \\
\text { esté atravesando un mal momento }\end{array}$ & 86,502 &, 000 & \\
\hline & $\begin{array}{l}\text { No me importa lo que los demás } \\
\text { piensen de mí }\end{array}$ & 47,758 &, 000 & \\
\hline & $\begin{array}{l}\text { Me cuesta mucho reponerme de } \\
\text { una separación emocional }\end{array}$ & 34,234 &, 025 & \\
\hline & Me considero una persona íntegra & 26,456 &, 033 & \\
\hline & $\begin{array}{l}\text { Siempre digo todo lo que pienso } \\
\text { aunque eso me perjudique }\end{array}$ & 50,481 & ,000 & \\
\hline & $\begin{array}{l}\text { Suelo considerar desagradable e } \\
\text { insoportable cualquier situación } \\
\text { estresante }\end{array}$ & 43,110 & ,002 & \\
\hline \multirow{3}{*}{$\begin{array}{l}\mathrm{N}^{\mathrm{o}} \text { Hijos } \\
\text { tres ítems } \\
\text { con valor } \mathrm{p}<, 05\end{array}$} & Me preocupo en exceso & 39,668 & ,001 & \multirow{3}{*}{$\begin{array}{l}\text { Con hijos }>\text { sin } \\
\text { hijos }\end{array}$} \\
\hline & $\begin{array}{l}\text { Tras una discusión me siento } \\
\text { hundido y vacío }\end{array}$ & 28,470 &, 028 & \\
\hline & $\begin{array}{l}\text { Siempre digo todo lo que pienso } \\
\text { aunque eso me perjudique }\end{array}$ & 34,729 & ,004 & \\
\hline
\end{tabular}


Si se tienen en cuenta las variables que se relacionan con el estatus profesional, encontramos que según sea el tipo de relación contractual, se dan diferencias en resiliencia que arrojan valores significativamente superiores, en las que el profesorado funcionario puntúa por encima de los y las docentes con contrato, en situación de interinidad y que realizan sustituciones. Por último, existen diferencias significativas en resiliencia en función de la antigüedad en el ejercicio docente en profesores y profesoras con más de 20 años de experiencia. En la Tabla 3, se expone de forma detallada las puntuaciones en resiliencia en relación a variables sociolaborales delcolectivo participante.

Tabla 3. Resiliencia en función de variables sociolaborales en docentes de E. Secundaria de Huesca.

\begin{tabular}{|c|c|c|c|c|}
\hline $\begin{array}{c}\text { Estatus } \\
\text { profesiona } \\
\text { l } \\
\end{array}$ & $\begin{array}{c}\text { Ítem } \\
\text { Resiliencia }\end{array}$ & $\mathrm{Chi}^{2}$ & $\mathbf{p}$ & Diferencias \\
\hline \multirow{8}{*}{$\begin{array}{l}\text { Relación } \\
\text { contractual } \\
\text { catorce } \\
\text { ítems } \\
\text { con valor } \\
\mathrm{p}<0,05\end{array}$} & $\begin{array}{l}\text { Miro al futuro } \\
\text { con optimismo } \\
\text { aunque esté } \\
\text { atravesando un } \\
\text { mal momento }\end{array}$ & $\begin{array}{c}16,91 \\
7\end{array}$ & $\begin{array}{c}, 05 \\
0\end{array}$ & \multirow{8}{*}{$\begin{array}{l}\text { Funcionario }>\text { Interino }>\text { Contratado }>\text { Sustit } \\
\text { uto }\end{array}$} \\
\hline & $\begin{array}{l}\text { Opino que es } \\
\text { bueno } \\
\text { aprender de los } \\
\text { errores }\end{array}$ & $\begin{array}{c}21,64 \\
9\end{array}$ & $\begin{array}{c}, 00 \\
1\end{array}$ & \\
\hline & $\begin{array}{l}\text { En ocasiones } \\
\text { me gustaría ser } \\
\text { otra persona }\end{array}$ & $\begin{array}{c}23,27 \\
3\end{array}$ & $\begin{array}{c}, 02 \\
5\end{array}$ & \\
\hline & $\begin{array}{l}\text { Soy } \\
\text { perseverante } \\
\text { en lograr mis } \\
\text { metas }\end{array}$ & $\begin{array}{c}18,55 \\
2\end{array}$ & $\begin{array}{c}, 02 \\
9\end{array}$ & \\
\hline & $\begin{array}{l}\text { Siento miedo } \\
\text { de que los } \\
\text { demás me } \\
\text { rechacen y me } \\
\text { quede solo }\end{array}$ & $\begin{array}{c}37,11 \\
4\end{array}$ & $\begin{array}{c}, 00 \\
\mathbf{0}\end{array}$ & \\
\hline & $\begin{array}{l}\text { Me dejo llevar } \\
\text { por las ideas } \\
\text { establecidas } \\
\text { socialmente }\end{array}$ & $\begin{array}{c}64,29 \\
9\end{array}$ & $\begin{array}{c}\mathbf{0 0} \\
\mathbf{0}\end{array}$ & \\
\hline & $\begin{array}{l}\text { Veo las cosas } \\
\text { blancas o } \\
\text { negras, pero } \\
\text { nunca veo el } \\
\text { término medio }\end{array}$ & $\begin{array}{c}26,12 \\
3\end{array}$ & $\begin{array}{c}, 01 \\
0\end{array}$ & \\
\hline & $\begin{array}{l}\text { No me importa } \\
\text { lo que los } \\
\text { demás piensen } \\
\text { de mí }\end{array}$ & $\begin{array}{c}40,81 \\
5\end{array}$ & $\begin{array}{c}\mathbf{0 0} \\
\mathbf{0}\end{array}$ & \\
\hline
\end{tabular}




\begin{tabular}{|c|c|c|c|c|}
\hline & $\begin{array}{l}\text { No me suelen } \\
\text { afectar las } \\
\text { críticas ni } \\
\text { positiva ni } \\
\text { negativamente }\end{array}$ & $\begin{array}{c}42,33 \\
8\end{array}$ & $\begin{array}{c}, 00 \\
0\end{array}$ & \\
\hline & $\begin{array}{l}\text { Ante cualquier } \\
\text { contratiempo } \\
\text { me siento } \\
\text { inseguro y creo } \\
\text { que no voy a } \\
\text { ser capaz de } \\
\text { soportarlo }\end{array}$ & $\begin{array}{c}31,59 \\
4\end{array}$ & $\begin{array}{c}, 00 \\
2\end{array}$ & \\
\hline & $\begin{array}{l}\text { No sé hacer } \\
\text { frente a } \\
\text { situaciones } \\
\text { difíciles }\end{array}$ & $\begin{array}{c}30,58 \\
8\end{array}$ & $\begin{array}{c}, 00 \\
2\end{array}$ & \\
\hline & $\begin{array}{l}\text { Tiendo a } \\
\text { exagerar los } \\
\text { acontecimiento } \\
\text { s negativos que } \\
\text { me ocurren }\end{array}$ & $\begin{array}{c}33,26 \\
0\end{array}$ & $\begin{array}{c}, 00 \\
1\end{array}$ & \\
\hline & $\begin{array}{l}\text { Soy de los que } \\
\text { piensa que en } \\
\text { todo lo malo } \\
\text { hay algo bueno }\end{array}$ & $\begin{array}{c}27,49 \\
4\end{array}$ & $\begin{array}{c}, 00 \\
7\end{array}$ & \\
\hline & $\begin{array}{l}\text { Suelo } \\
\text { considerar } \\
\text { desagradable e } \\
\text { insoportable } \\
\text { cualquier } \\
\text { situación } \\
\text { estresante }\end{array}$ & $\begin{array}{c}22,71 \\
5\end{array}$ & $\begin{array}{c}, 03 \\
0\end{array}$ & \\
\hline \multirow{3}{*}{$\begin{array}{l}\text { Años de } \\
\text { docencia } \\
\text { nueve } \\
\text { ítems } \\
\text { con valor } \\
\mathrm{p}<0,05\end{array}$} & $\begin{array}{l}\text { Tras una } \\
\text { discusión me } \\
\text { siento hundido } \\
\text { y vacío }\end{array}$ & $\begin{array}{c}36,74 \\
1\end{array}$ & $\begin{array}{c}, 00 \\
2\end{array}$ & \multirow{3}{*}{$\begin{array}{l}\text { Más de } 20 \text { años de ejercicio docente }>\text { de } 5 \\
\text { a } 15 \text { años }>\text { de } 15 \text { a } 20 \text { años }>\text { de } 3 \text { a } 5 \\
\text { años }>\text { menos de tres años }\end{array}$} \\
\hline & $\begin{array}{l}\text { Cuando recibo } \\
\text { una mala } \\
\text { noticia tardo } \\
\text { días e incluso } \\
\text { meses hasta } \\
\text { que me } \\
\text { repongo }\end{array}$ & $\begin{array}{c}25,91 \\
3\end{array}$ & $\begin{array}{c}, 05 \\
5\end{array}$ & \\
\hline & $\begin{array}{l}\text { Suelo } \\
\text { considerar } \\
\text { desagradable e }\end{array}$ & $\begin{array}{c}27,19 \\
1\end{array}$ & $\begin{array}{l}, 03 \\
9\end{array}$ & \\
\hline
\end{tabular}




\begin{tabular}{|c|c|c|}
\hline $\begin{array}{l}\text { insoportable } \\
\text { cualquier } \\
\text { situación } \\
\text { estresante }\end{array}$ & & \\
\hline $\begin{array}{l}\text { Me enfrento a } \\
\text { las situaciones } \\
\text { estresantes sin } \\
\text { dejar que me } \\
\text { dominen las } \\
\text { situaciones. }\end{array}$ & $\begin{array}{c}27,22 \\
2\end{array}$ & $\begin{array}{c}, 00 \\
7\end{array}$ \\
\hline $\begin{array}{l}\text { Suelo llevar } \\
\text { mal } \\
\text { equivocarme }\end{array}$ & $\begin{array}{c}54,97 \\
2\end{array}$ & $\begin{array}{c}, 00 \\
7\end{array}$ \\
\hline $\begin{array}{l}\text { Tras una } \\
\text { discusión me } \\
\text { siento hundido } \\
\text { y vacío. }\end{array}$ & $\begin{array}{c}56,71 \\
0\end{array}$ & $\begin{array}{c}, 00 \\
5\end{array}$ \\
\hline $\begin{array}{l}\text { No me importa } \\
\text { lo que los } \\
\text { demás piensen } \\
\text { de mí }\end{array}$ & $\begin{array}{c}71,41 \\
6\end{array}$ & $\begin{array}{c}, 00 \\
\mathbf{0}\end{array}$ \\
\hline $\begin{array}{l}\text { No me suelen } \\
\text { afectar las } \\
\text { críticas ni } \\
\text { positiva ni } \\
\text { negativamente. }\end{array}$ & $\begin{array}{c}67,74 \\
6\end{array}$ & $\begin{array}{c}, 00 \\
\mathbf{0}\end{array}$ \\
\hline $\begin{array}{l}\text { Soy de los que } \\
\text { piensa que en } \\
\text { todo lo malo } \\
\text { hay algo bueno }\end{array}$ & $\begin{array}{c}63,64 \\
3\end{array}$ & $\begin{array}{c}, 00 \\
1\end{array}$ \\
\hline
\end{tabular}

\section{DISCUSIÓN}

Los datos reflejan que se trata de un colectivo experimentado, destacando la baja participación del profesorado novel y con contrato eventual frente al profesorado funcionario que se muestra más colaborativo. Igualmente, resalta la participación mayoritaria por parte de la mujer, así como la mayor colaboración del profesorado casado y con hijos.

En cuanto a los niveles de resiliencia, parece que tiende a desarrollarse más en las mujeres que en los hombres, aspecto que puede estar en la base de su afrontamiento de adversidades en el ámbito laboral, inherentes al trabajo docente en la etapa secundaria. Stratta et al. (2013) comparan niveles de resiliencia encontrando diferencias significativas en las fuentes de resiliencia entre varones y mujeres, siendo mayor en las mujeres, al igual que ocurre en este estudio. Serrano (2018) en una investigación con triatletas, reporta que las mujeres resultan ser más resistentes que los hombres, si bien el autor explica que estas diferencias no son estadísticamente significativas.

Según el rango de edad, las puntuaciones más altas en resiliencia se obtienen en la franja de 46 


\section{EMOCIONES POSITIVAS: UNA HERRAMIENTA PSICOLÓGICA QUE CONTRIBUYE AL PROCESO DE RESILIENCIA EN LOS PROFESIONALES DE LA EDUCACIÓN}

a 55 años. Sin embargo, Saavedra y Villalta (2008), en un estudio comparativo en personas entre 15 y 65 años concluyen que los niveles de resiliencia no están relacionados directamente a los tramos de edad y solo aprecian una diferencia significativa entre los 19 y 24 años, con puntajes más altos y entre 46 y 55 años en el que obtienen los más bajos. Por su parte, Álvarez y Cáceres (2010) en una investigación sobre resiliencia y variables sociodemográficas concluyen que la edad no hace más o menos resiliente a una persona; es decir, puede haber adultos muy poco resilientes o jóvenes muy resilientes.

El profesorado con estado civil casado o viviendo en pareja presenta mayores características resilientes frente al soltero, por lo que se identifica como un factor de protección en términos de resiliencia. Albar et al. (2004) defienden que la tenencia de hijos/as funciona como factor de protección frente a los estresores, lo que se relaciona con el apoyo emocional recibido por parte de la familia. En sentido contrario, Justo (2010) confirma que los problemas familiares que distorsionan el funcionamiento normal de la dinámica del hogar, aumentan la vulnerabilidad del sujeto frente a los estresores.

Las puntuaciones más altas en resiliencia se obtienen en el Factor I, sensibilidad o afrontamiento emocional, Factor III positivismo u optimismo ingenuo y Factor II, superación 0 afrontamiento conductual, respectivamente.

Los resultados reportados en el Factor I confirman los trabajos de Ornelas (2016) Contreras y Peña (2016) y Carrara (2018) quienes puntualizan que estrategias inadecuadas, de tipo escapista, aumentan la vulnerabilidad a los estresores laborales. Este es un dato negativo, que pone de manifiesto que parte del profesorado encuestado responde emocionalmente ante los estresores.

Las valoraciones encontradas en el Factor II son muy positivas y corroboran los estudios de Knowlden et al. (2016), González et al. (2017), Alarcón (2018) y Vicente de Vera y Gabari (2019), entre otros, quienes subrayan que algunas personas son más resistentes a los estresores, lo que les posibilita poner en marcha estrategias de afrontamiento para superar las demandas que les genera estrés. Por tanto, los resultados de este componente demuestran la gran capacidad del colectivo encuestado para hacer frente a los obstáculos laborales.

En el Factor III se constatan las aportaciones de McKergow (2009), Carver y Scheier (2014), Gómez y Cavaco (2016) y Díaz y Barra (2017) cuando defienden que las emociones positivas provocan, fundamentalmente, cambios en la actividad cognitiva y posteriormente cambios en la esfera conductual. Esto favorece la construcción de recursos personales (físicos, psicológicos y sociales) para afrontar las situaciones difíciles o problemáticas, que propician un estilo de afrontamiento adaptativo o resiliente (Morales, 2017). Existe consenso en defender la estrecha relación entre optimismo y resiliencia (Dawson y Pooley, 2013; Martínez y Ruch, 2017). La experiencia de emociones positivas no es más que el reflejo de un modo resiliente de afrontar las situaciones adversas (Santos, 2013; Oriol et al., 2017; Félix et al., 2018). Por el contrario, una actitud pesimista ante los estresores hace que las personas se manifiesten dudosas y vacilantes (Pulido y Herrera, 2018).

\section{CONCLUSIONES}

Se da relación entre el sexo y el desarrollo de resiliencia, siendo las mujeres las que manifiestan más características resilientes frente a los hombres.

Algunas variables sociodemográficas actúan como factores protectores o promotores de resiliencia, como son: el estado civil que conlleva acompañamiento (por matrimonio o pareja formalizada); la presencia de descendencia; la etapa vital entre los 46 y 55 años; el status laboral estable de funcionario; y la antigüedad de más de 20 años de ejercicio docente.

La actitud optimista se relaciona con la confianza y la persistencia en la conducta, lo que tiene 
consecuencias en la forma de afrontar los cambios, la adversidad y la experiencia de estrés en el trabajo. Las emociones positivas son un elemento efectivo ante la adversidad favorecedores del surgimiento de la resiliencia del colectivo encuestado. En la medida que refuerzan estos atributos, mitigan la vulnerabilidad a los estresores laborales.

Algunas limitaciones son, en primer lugar, la muestra utilizada representa un colectivo muy específico, profesores de Institutos públicos de la provincia de Huesca. En futuros trabajos se debería tratar de acceder a una muestra más amplia y que incluya docentes de todos los niveles y etapas, así como a otros centros educativos a nivel nacional en diversas comunidades autónomas, para poder hacer estudios comparativos.

A pesar de las limitaciones citadas, los resultados presentados tienen importantes implicaciones prácticas de cara a facilitar el bienestar del profesorado de Secundaria. Este estudio aporta nueva información sobre la vinculación existente entre la resiliencia y el optimismo, que puede contribuir al diseño de programas eficaces para la prevención del estrés en el colectivo docente. Dichos programas deberían incluir estrategias que fomenten el desarrollo de características psicológicas como la resiliencia y el optimismo. Los desafíos a futuros han de coger este testigo para crear las condiciones necesarias para que este colectivo encuentre caminos de desarrollo profesional de menor coste personal.

\section{REFERENCIAS BIBLIOGRÁFICAS}

Alarcón, R. (2018). La formación para el trabajo y el paradigma de formación por competencias. Calidad en la Educación, (16), 143-156.

Albar, M. J., Romero, M. E., González, M. D., Carbayo, E., García, A., Gutiérrez, I. \& Algaba, S. (2004). Apoyo social, características sociodemográficas y burnout en enfermedas y auxiliares del hospital. Enfermería Clínica, 14(5), 281-285. doi.org/10.1016/S1130-8621(04)73899-1

Álvarez, L. Y. \& Cáceres, L. (2010). Resiliencia, rendimiento académico y variables sociodemográficas en estudiantes universitarios de Bucaramanga (Colombia). Psicología Iberoamericana, 18(2).

Carrara, J. L. (2018). Personalidad resistente y su relación con el egreso voluntario en call centers. Revista de Psicología, 36(1), 239-263. doi.org/10.18800/psico.201801.009

Carver, C. S. \& Scheier, M. F. (2014). Dispositional optimism. Trends in cognitivesciences, 18(6), 293-299.

Contreras, M. A. \& Peña, A. I. B. (2016). Estrés y burnout en profesores de primaria y secundaria de Huelva: las estrategias de afrontamiento como factor de protección. International Journal of Developmental and Educational Psychology. Revista INFAD de Psicología., 4(1), 303-310. doi.org/10.17060/ijodaep.2014.n1.v4.616

Dawson, M. \& Pooley, J. A. (2013). Resilience: The Role of Optimism, Perceived Parental Autonomy Support and Perceived Social Support in First Year University Students. Journal of Education and Training Studies, 1(2), 38-49. doi: 10.11114/jets.v1i2.137

Díaz, C. \& Barra, E. (2017). Resiliencia y satisfacción laboral en profesores de colegios municipales y particulares subvencionados de la comuna de Machalí. Estudios pedagógicos (Valdivia), 43(1), 75-86. doi.org/10.4067/S0718-07052017000100005

Félix, R. 0., García, C. \& Mercado, S. M. (2018). El estrés en el entorno laboral. Revisión genérica desde la teoría. CULCYT, (64), 31-42.

Franco, G. (2017). Inteligência emocional como fator protetor do burnout em professores do 2. ${ }^{\circ} \mathrm{e}$ 3. ․ ciclos e secundário da ram. International Journal of Developmental and Educational Psychology. Revista INFAD de Psicología., 6(1), 417- 


\section{EMOCIONES POSITIVAS: UNA HERRAMIENTA PSICOLÓGICA QUE CONTRIBUYE AL PROCESO DE RESILIENCIA EN LOS PROFESIONALES DE LA EDUCACIÓN}

426.doi.org/10.17060/ijodaep.2014.n1.v6.761

Gómez, M. C. A. \& Cavaco, N. A. A. P. (2016). La resiliencia del docente como factor crucial para superar las adversidades en una sociedad de cambios. Tendencias pedagógicas, 28(1), 167-180.

González, T. M. P., Torres, L. H. \& Carreres, A. L. (2017). Fortalezas y virtudes personales del profesorado y su relación con la eficacia docente. International Journal of Developmental and Educational Psychology (Revista INFAD de Psicología), 7(1), 141-150. doi.org/10.17060/ijodaep.2014.n1.v7.785

Justo, C. F. (2010). Intervención sobre los niveles de burnout y resiliencia en docentes de educación secundaria a través de un programa de conciencia plena (mindfulness) Revista Complutense de Educación, 21(2), 271-288.

Knowlden, A.P., Hackman, C.L. \& Sharma, M. (2016). Lifestyle and mental health correlates of psychological distress in college students. Health Education Journal, 75(1), 370-382. doi.org/10.1177/0017896915589421

Kristjánsson, K. (2012). Positive psychology and positive education: Old wine in new bottles? Educational Psychologist, 47(2), 86-105. doi.org/10.1080/00461520.2011.610678

Martínez, M. L. \& Ruch, W. (2017). Character strengths predict resilience over and above positive affect, self-efficacy, optimism, social support, self-esteem, and life satisfaction. The Journal of Positive Psychology, 12(2), 110-119. doi.org/10.1080/17439760.2016.1163403

Martínez, S. M. (2010). Construcción y análisis psicométrico de un Cuestionario de evaluación de la resiliencia. Recuperado de: http://www. unizar. es/abarrasa/tea/200910_25906/serrano2010.pdf.

Masten, A. S. (2016). Resilience in developing systems: the promise of integrated approaches. European Journal of Developmental Psychology, 13, 297-312. doi.org/ 10.1080/17405629.2016.1147344

McKergow, M. (2009). Positivity: Groundbreaking research reveals how to embrace the hidden strengths of positive emotions, overcome negativity and thrive. InterAction-The Journal of Solution Focus in Organisations, 1(1), 89-92.

Morales, F. M. (2017). Relaciones entre afrontamiento del estrés cotidiano, autoconcepto, habilidades sociales e inteligencia emocional. European Journal of Education and Psychology, 10(1), 4148. doi.org/10.1016/j.ejeps.2017.04.001

Oriol, X., Mendoza, M., Covarrubias, C. G. \& Molina, V. M. (2017). Emociones positivas, apoyo a la autonomía y rendimiento de estudiantes universitarios: el papel mediador del compromiso académico y la autoeficacia. Revista de Psicodidáctica, 22(1), 45-53. doi.org/10.1016/S11361034(17)30043-6

Ornelas, P. E. (2016). Estrategias de afrontamiento y resiliencia en cuidadores primarios con duelo. RevistaPsicología y Salud, 26(2), 177-184.

Pulido, F. \& Herrera, F. (2018). Predictors of Happiness and Emotional Intelligence in Secondary Education. Revista Colombiana de Psicología, 27(1), 71-84.doi.org/10.15446/rcp.v27n1.62705

Saavedra, E. \& Villalta, M. (2008). Escala de Resiliencia SV-RES, para jóvenes y adultos. Santiago: Ceanim.

Serrano, J. (2018). Resiliencia, optimismo y burnout en triatletas de un club deportivo de running, trail y triathlon. Recuperado de:http://hdl.handle.net/11201/147817

Stratta, P., Capanna, C., Patriarca, S., de Cataldo, S., Bonanni, R. L., Riccardi, I. \& Rossi, A. (2013). Resilience in adolescence: gender differences two years after the earthquake of L'Aquila. Personality and Individual Differences, 54(3), 327-331.doi.org/10.1016/j.paid.2012.09.016 Vicente de Vera, M. I. \& Gabari, M. I. (2019). Liderazgo Pedagógico en Educación Secundaria: 
Aportaciones desde la Evaluación de Burnout-Resiliencia en Docentes. International Journal of Educational Leadership and Management, 7(1), 104-134. doi.org/10.17583/ijelm.2018.3519

La presente investigación no ha recibido ninguna beca específica de agencias de los sectores público, comercial, o sin ánimo de lucro. 
